\title{
High titres of immunoglobulin g class 1 antibodies to duffy binding-like (DBL) 5 during the third trimester of pregnancy are associated with past placental malaria at delivery in an area of high seasonal transmission in Burkina Faso.
}

Ousmane Traoré ${ }^{1,2}$, Kaboré W. O. Benjamin ${ }^{1}$, Esmée Ruizendaal ${ }^{8}$, Hermann Sorgho ${ }^{1}$, Innocent Valéa ${ }^{1,3}$, Maminata Traoré-Coulibaly', Susana Scott ${ }^{7}$, Petra F. Mens ${ }^{5}$, Henk D. F. H. Schallig', Yves Traorée, Adrien M. G. Belem ${ }^{2}$, Umberto D’Alessandro ${ }^{6}$ and Halidou Tinto ${ }^{1}$

${ }^{1}$ Institut de Recherche en Sciences de la Santé, Unité de Recherche Clinique de Nanoro (IRSS-URCN), Nanoro, Burkina Faso

${ }^{2}$ Université Nazi Boni de Bobo-Dioulasso (UNB), Bobo-Dioulasso, Burkina Faso

${ }^{3}$ Centre National de Recherche et de Formation sur le Paludisme (CNRFP), Ouagadougou, Burkina Faso

${ }^{4}$ Université Ouaga I, Pr Joseph Ki-Zerbo, Ouagadougou, Burkina Faso

${ }^{5}$ Amsterdam University Medical Centers- Academic Medical Centre, Department of Medical Microbiology,

Parasitology Unit, Amsterdam, The Netherlands

${ }^{6}$ Medical Research Council Unit the Gambia at the London School of Hygiene and Tropical Medicine, The Gambia

${ }^{7}$ Department of Infectious Disease Epidemiology, London School of Hygiene and Tropical Medicine, London, UK

${ }^{8}$ Department of Medical Microbiology, University Medical Center Utrecht, Utrecht, The Netherlands

\begin{abstract}
Objective: In malaria endemic area, women acquire throughout successive pregnancies, increasing protection against Plasmodium falciparum malaria infections by having increased levels of antibodies to the Variant 2 Cell Surface antigen- Chondroitin Sulphate A. Primigravidae are more susceptible to placental malaria due to the lack of these specific antibodies to placental malaria-antigens. However, it is currently difficult to diagnose placental malaria by a noninvasive method prior to delivery. The objective of this study was to assess whether VAR2CSA specific antibody levels from peripheral blood obtained during the third trimester of pregnancy, could predict placental infection at delivery.
\end{abstract}

Methods: A total of 263 pregnant women participating to the COSMIC study (NCT01941264) were included in this study. Peripheral blood samples were collected at the third antenatal care visit performed during the third trimester of pregnancy. Placental infec-tions were determined by histology. Antibody levels (total IgG, IgG1 and IgG3) to VAR2CSA subdomains DBL5 and ID1-ID2a in serum samples were quantified using En-zyme Linked ImmunoSorbent Assay.

Results: Placental malaria was estimated at $54.9 \%$ among pregnant women enrolled in the study. More than half of the study population had a past placental infection. Among factors investigated, in univariate analyses ITN use, gravidity, season of delivery, number of IPTp-SP doses, gestational age and peripheral malaria status at the 3rd ANC visit significantly affected DBL5 and ID1ID2a specific antibody titres. In multivariate analyses, ITN use (OR=5.17, 95\% CI: $(1.36$ - 19.61), $p=0.016)$ was associated with the risk of placental malaria at delivery, while older women were at lower risk $(\mathrm{OR}=0.45,95 \% \mathrm{CI}:(\mathbf{0 . 2 3 - 0 . 8 8}), \mathrm{p}=\mathbf{0 . 0 2 1})$. Importantly, higher levels of IgG1 anti-DBL5 at the 3rd trimester $(\mathrm{OR}=11.96,95 \%$ CI: $(3.69-38.77), \mathrm{p}<0.001)$ were significantly associated with placental malaria.

Conclusions: Findings of this study indicate that high levels of DBL5 IgG1 at the third tri-mester of pregnancy could predict placental malaria, which was mostly past infections in our study population. The assessment of this exposition marker during pregnancy could alterna-tively be used as an optional biomarker for PM diagnosis.

Keywords: IgG1, IgG3, DBL5, ID1-ID2a, Placental malaria.

\section{Introduction}

Over the last 20 years, several preventive interventions such as Long-Lasting Insecticidal Nets (LLIN), intermittent preventive treatment for pregnant women [1] and Seasonal Malaria Chemoprevention (SMC) for children 3-59 months living in areas of seasonal malaria transmission [2], have been scaled up in malaria-endemic countries [3], resulting in a significant reduction of the malaria burden worldwide. Nevertheless, in 2016 the number of malaria cases was estimated at 212 million, 
Citation: Traoré O, Benjamin Kaboré WO, Ruizendaal E, Sorgho H, Valéa I, et al. High titres of immunoglobulin g class 1 antibodies to duffy binding-like (dbl) 5 during the third trimester of pregnancy are associated with past placental malaria at delivery in an area of high seasonal transmission in burkina faso. Res Rep Immunol. 2018;2(1):11-2

resulting in 429,000 deaths [4]. Besides children under 5 years old, pregnant women remain one of the most vulnerable groups $[3,5]$. Malaria during pregnancy results in parasite sequestration in the placenta, i.e. placental malaria (PM), which in turn causes intra-uterine growth retardation and low birth weight, a risk factor for infant mortality [6]. P. falciparum parasites sequester in the placenta thanks to binding between the PfEMP1 variant 2 cell surface antigen-chondroitin sulfate A (VAR2CSA) at the surface of the infected erythrocytes and the placenta's chondroitin sulfate A (CSA) receptor [7-10]. Women's immunity against parasites enables to sequester in the placenta, develops during subsequent pregnancies as shown by the higher antibody levels to at least one VAR2CSA domain or to the fulllength protein in multigravida as compared to primigravidae [10-12]. Because malaria infection may increase VAR2CSA antibody levels and thereby induce confounding in the analyses of immune protection [13], it is actually necessary to assess also the effect of factors that can influence antibody levels.

The presence of highly conserved residues in surface-exposed areas of the VAR2CSA suggests that the DBL5 domain is immune-dominant as confirmed by the higher antibody levels to this domain in multigravidae; parity-dependent reactivity to other domains is less consistent [14]. Antibodies against the recombinant DBL5 domain amplified from placental parasites bind native VAR2CSA from placental isolates [15]. In rat models, antibodies to a fragment of VAR2CSA, composed by the interdomain 1 (ID1), the Duffy Binding like 2 (DBL2) domain and the ID2 (combined and known as ID1-ID2a), inhibited binding of infected erythrocytes [15]. Though cytophilic antibodies (IgG1 and $\operatorname{IgG} 3$ ) are the main subclasses involved in acquired VSAPAM-specific immunity to pregnancy-associated malaria [16], little is known about their ability to predict placenta infections. The aim of this study was to analyze the profile of antibody to DBL5 and ID1-ID2a VAR2CSA subdomains during the third trimester of pregnancy and to assess whether these antibodies could predict PM at delivery.

\section{Materials and Methods}

\section{Study setting}

This study was conducted in the Nanoro Health and Demographic Surveillance System (HDSS) catchment area, located at $85 \mathrm{~km}$ from Ouagadougou, the capital city of Burkina Faso. This is an area of intense and seasonal malaria transmission, occurring from July to December. During this period, malaria represents almost half (48.6\%) of all outpatient consultations. PM prevalence as determined by molecular methods is estimated at $34.6 \%[17]$.

\section{Study design}

The present study was nested within a large trial investigating the efficacy of different preventive strategies against malaria in pregnancy [Community-based scheduled screening and treatment of malaria in pregnancy for improved maternal and infant health: a cluster-randomized trial (COSMIC)] whose protocol has been described elsewhere [18]. Briefly, the COSMIC study aimed to determine whether adding to standard IPTp-SP monthly malaria screening and treatment (CSST) at village level by Community Health Workers (CHWs) would further reduce placental malaria compared to IPTp-SP alone. However, only women in the control arm, i.e. who received only standard IPTp-SP, were included in this study.

\section{Sample collection}

Approximately $2 \mathrm{ml}$ of peripheral blood was collected in heparin tubes from each participant at ANC 3 visit and who was in the $3^{\text {rd }}$ trimester of her pregnancy. The samples were transferred to the Clinical Research Unit of Nanoro (CRUN) laboratory where they were processed within 4 hours after sampling. Blood slides were first prepared, and subsequently, the remaining blood was centrifuged at $500 \mathrm{rpm}$ for 10 minutes and the collected serum was stored at $-80^{\circ} \mathrm{C}$. At the time of delivery, a $2 \mathrm{~cm} \times 2 \mathrm{~cm} \times 1 \mathrm{~cm}$ placental biopsy sample was collected into $10 \%$ neutral buffered formalin and later embedded in paraffin wax for histological analysis. Maternal peripheral blood at delivery was also taken for hemoglobin $(\mathrm{Hb})$ measurement using a Hemocue $(\mathrm{Hb} 301$, Sweden); anemia was defined as $\mathrm{Hb} \leq 1 \mathrm{~g} / \mathrm{dl}$.

\section{Microscopy}

Blood slides prepared during the ANC 3 visit, were stained with Giemsa 3\% for 45-60 minutes. Slides were read by two independent microscopists blinded to the rapid diagnostic test (RDT) results. Parasite density was calculated against 200 leukocytes, or against 500 leukocytes if the count was $<10$ parasites/200 leukocytes. Slides were considered negative if no parasites were seen after examination of 100 high power fields. Any discrepancy between the two readings was resolved by a third independent reader.

\section{Antigens and antibody levels measurement by ELISA}

The recombinant antigens (DBL5 and ID1-ID2a) were produced [19] and freely offered by Professor Ali Salanti (Faculty of Health Sciences, University of Copenhagen, Denmark). The DBL5 domain $(50 \mathrm{kD})$ from FCR3 was produced in insect cells purified by Histidine-Tagged protein (HIS tag) purification procedure, while the ID1-ID2a fragment $(75 \mathrm{kD})$ from FCR3 was produced in Escherichia coli purified by Ion exchange (IEX) protocol. Both HIS tag purification and IEX method were followed by size exclusion procedure, as described by Clausen et al. [19].

For the serological analysis, optimal concentrations of each protein were coated in 96-well microtitration plates, and the different subtypes of specific IgG were measured by ELISA following the method described by Guitard et al [20]. Briefly, the plates were coated with $1 \mu \mathrm{g} / \mathrm{ml}$ of either DBL5-domain or ID1-ID2a domain overnight and then incubated with $100 \mu \mathrm{l}$ of human plasma at 1:100 dilutions. The secondary antibody was a horseradish peroxidase-conjugated anti-human IgG (Life Technology, A24470) for total IgG measures. Between each step, the plates were washed with PBS-Tween ${ }^{\mathrm{TM}} 20$ buffer at $0.5 \%$. For the IgG subclasses, purified mouse monoclonal antibodies against human IgG1 and IgG3 (Life Technology, A10648, MH1732) were used. All reagents were used at predetermined optimal dilution (IgG: 1:10,000; IgG1: 1: 2,000 and IgG3: 1: 5000 respectively). Pooled serum samples from hyper-immune multigravid women living in Nanoro were used as a positive control; these samples were previously tested against DBL5 
Citation: Traoré O, Benjamin Kaboré WO, Ruizendaal E, Sorgho H, Valéa I, et al. High titres of immunoglobulin g class 1 antibodies to duffy binding-like (dbl) 5 during the third trimester of pregnancy are associated with past placental malaria at delivery in an area of high seasonal transmission in burkina faso. Res Rep Immunol. 2018;2(1):11-2

and ID1-ID2a to ensure that they were high responders to these antigens. Negative control sample comprised pooled serum samples from Caucasian male donors not exposed to malaria infection. The optical density (OD) was obtained by subtracting the average OD of duplicate wells from that of the corresponding blank wells using ELISA-Reader Multiskan TM GO's software. Seropositivity was defined as antibody levels (in OD values) $>2$ standard deviations (SD) above the mean absorbance yielded by the pooled serum samples from Caucasian donors and seronegative $\mathrm{OD}$ values $\leq 2 \mathrm{SD}$.

\section{Placental malaria}

Slides from the placenta biopsies obtained from the maternalfacing side were stained with hematoxylin-eosin [18] and read by trained microscopists. Placental infection was classified as follows:

(1) Acute infection (parasites present, malaria pigment absent)

(2) Chronic infection (parasites and malaria pigment present)

(3) Past infection (no parasites but pigment present)

(4) No infection (both parasites and malaria pigment absent) [21]

\section{Statistical analysis}

The distributions of continuous variables were assessed for normality by using the Shapiro-Wilks W test, and non-normal continuous data were log-transformed. Spearman's rho test was used to assess correlations between continuous variables. To evaluate the difference between different groups generated by stratifying the study population according to the following variables: use of ITN, anemia, gravidity, the season of delivery, age, IPTp-SP, gestational age and malaria status at ANC3. The antibody levels to DBL5 and ID1-ID2a were compared using the Mann-Whitney test. A simple linear regression analysis (bivariate) was performed to assess the association between transmission season, gravidity, malaria status at the third antenatal care (ANC) visit as determined by microscopy, number of IPTp-SP doses, gestational age, women's age and antibody levels to DBL5 and ID1-ID2a. Then, the adjustment was performed in a multiple linear regression (multivariate analysis), including all the variables. In addition, in a logistic regression, bivariate-analyses were performed (including all variables and all the antibody types (IgG, IgG1 and $\mathrm{IgG} 3$ ) to DBL5 and ID1-ID2a) and multivariate logistic regression (including all variables significantly associated with PM and all antibody types significantly associated with PM) to build an explanatory model for PM and to look for confounding, interaction and linear trends. Values of $\mathrm{p}<0.05$ were considered as statistically significant. Statistical analyses were performed using Stata Statistical Software: Release 15 (College Station, TX).

\section{Results}

\section{Characteristics of the study population}

Two hundred sixty-three pregnant women were included in the study (Table 1). Their mean age was $25.6 \pm 5.9$ years and the majority of them $(83.3 \%)$ were multigravida (gravidity $\geq 2$ ); $77.2 \%$ of the study population had a gestational age of at least or above the median gestational age ( 32 weeks). At the third ANC visit, malaria peripheral infection was diagnosed by microscopy in $14.1 \%(37 / 263)$ of women and the median parasite densities detected was estimated at 733 [95\% CI: $0-58860.5]$ parasites $/ \mu \mathrm{L}$. At delivery, more than half of women had PM, although most of them had malaria pigment, without parasites. At delivery, anemia was reported in $17.1 \%(n=45 / 263)$ of women included in this study. IPTp-SP coverage was high as most of them had received at least 3 doses and the use of insecticide-treated bed net was also high (Table 1).

\section{Antibody profiles in pregnant women at the third $A N C$ visit}

To test the capability of naturally acquired antibodies to recognize VAR2CSA recombinant fragments, antibodies raised naturally against DBL5 and ID1-ID2a were quantified from the 263 pregnant women at their $3^{\text {rd }}$ ANC visit. Women were classified into two subgroups (seropositive and seronegative). The analysis of antibody profiles showed that $100 \%(263 / 263)$ of women were seropositive for DBL5 $\operatorname{IgG} 1,96.6 \%(\mathrm{n} / \mathrm{N}$ : 254/263) for total IgG and $30.8 \%$ for DBL5 IgG3. For ID1ID2a, while $48.3 \%$ (127/263) of seropositivity was found for IgG3, 100\% of pregnant women were seropositive for IgG1 and 94.7\% (249/263) for total IgG (Additional file 1). It appears that women living in the study area have naturally developed detectable antibodies that can specifically recognize VAR2CSA recombinant fragments (DBL5 and ID1-ID2a).

Table 1. Characteristics of study population.

\begin{tabular}{|c|c|}
\hline Characteristics & Value \\
\hline Age -years, Mean \pm SD & $25,57 \pm 5,89$ \\
\hline Age $\geq$ Mean -no. (\%) & $120(45.6)$ \\
\hline \multicolumn{2}{|l|}{ Gestational age (weeks) the 3rd ANC -no. (\%) } \\
\hline$\geq$ median age & $203(77.2)$ \\
\hline \multicolumn{2}{|l|}{ Gravidity -no. (\%) } \\
\hline Primigravidae & $44(16.8)$ \\
\hline Multigravidae & $219(83.3)$ \\
\hline Hemoglobin $(\mathrm{Hb})$ levels at delivery-g/dL Mean $\pm \mathrm{SD}$ & $12.1 \pm 1.5$ \\
\hline \multicolumn{2}{|l|}{ Anaemia at delivery $(\mathrm{Hb} \leq 11 \mathrm{~g} / \mathrm{dl})-\mathrm{no} .(\%)$} \\
\hline Anemic & $45(17.1)$ \\
\hline \multicolumn{2}{|l|}{ Placental infections at delivery } \\
\hline \multicolumn{2}{|l|}{$(\mathrm{N}=211)$-no. $(\%)$} \\
\hline Acute & $1(0.5)$ \\
\hline Chronic & $3(1.4)$ \\
\hline Past & $113(53.6)$ \\
\hline No infection & $94(44.6)$ \\
\hline \multicolumn{2}{|l|}{ Peripheral malaria at the 3rd ANC-no. (\%) } \\
\hline Non-infected & $226(85.9)$ \\
\hline Infected & $37(14.1)$ \\
\hline $\begin{array}{l}\text { Parasite density of peripheral infection at the 3rd ANC -Parasite/ } \\
\mu \mathrm{L}, \text { Median (IQR) }\end{array}$ & $\begin{array}{l}733(0- \\
58860.5)\end{array}$ \\
\hline \multicolumn{2}{|l|}{ IPTp-SP doses from recruitment to 3rd ANC-no. (\%) } \\
\hline$\leq 2$ doses & $177(67.3)$ \\
\hline \multicolumn{2}{|l|}{ ITN ownership before the 3rd ANC-no. (\%) } \\
\hline Yes & $245(93.2)$ \\
\hline No & $16(6.1)$ \\
\hline Other (Don't know) & $2(0.7)$ \\
\hline \multicolumn{2}{|l|}{ Used of an ITN the previous night } \\
\hline \multicolumn{2}{|l|}{$(\mathrm{N}=233)-\mathrm{n}(\%)$} \\
\hline Yes & $212(91)$ \\
\hline No & $21(9)$ \\
\hline \multicolumn{2}{|l|}{ Season of delivery-n (\%) } \\
\hline High transmission (July-December) & $147(55.9)$ \\
\hline Low transmission (January-June) & $116(44.1)$ \\
\hline
\end{tabular}


Citation: Traoré O, Benjamin Kaboré WO, Ruizendaal E, Sorgho H, Valéa I, et al. High titres of immunoglobulin g class 1 antibodies to duffy binding-like (dbl) 5 during the third trimester of pregnancy are associated with past placental malaria at delivery in an area of high seasonal transmission in burkina faso. Res Rep Immunol. 2018;2(1):11-2

This Supplemental Table 1 presents frequencies of seropositive and seronegative women to DBL5 and ID1-ID2a. Seropositive subjects are defined as antibody levels $>2$ standard deviations (SD) above the mean absorbance yielded by the pooled serum samples from Caucasian donors. Seronegative ones are defined as antibody levels $\leq 2 \mathrm{SD}$.

\section{Relationships between titers of antibody types}

DBL5 and ID1-ID2a are both two fragments of VAR2CSA antigen. To further check the antigens quality, the relationships between the titres of antibody types were determined, based on the graded interpretation of Spearman's correlation test (Additional file 2).

This Supplemental Table 2 presents results of the Spearman's correlation test coefficients aiming to assess the relationships between the titers of the different antibody types analyzed in this study. Spearman's correlation graded interpretation is defined as follows: (i) rho between $0.1-0.3=$ weak; (ii) rho between $0.4-0.7=$ moderate and (iii) rho between $0.8-1.0=$ strong correlation. Thus, IgG1 anti-DBL5 and IgG1 anti-ID1-ID2a on the one hand (Spearman's rho $=0.661, p<0.001$ ) and $\operatorname{IgG}$ antiDBL5 and IgG anti-ID1-ID2a (Spearman's rho $=0.423, \mathrm{p}<0.001$ ) on the other hand appeared to be moderately correlated. Only these two correlation plots have been presented in this manuscript. Titres of different antibody types were more often positively but weakly correlated, as rho values did not exceed the cut-off fixed between 0.1-0.3. Nevertheless, moderate correlations were found between IgG1 anti-DBL5 and IgG1 anti-ID1-ID2a ( $r h o=0.661, p<0.001)$ (Figure 1a) and between IgG anti-DBL5 and IgG anti-ID1-ID2a $(\mathrm{rho}=0.423, \mathrm{p}<0.001)$
(Figure1b).

\section{Assessment of the relationship between antibody titers at the $3^{\text {rd }}$ ANC and factors that influence antibody levels}

To analyze the effect of factors available in the dataset, linear regression was performed and correlation coefficients were reported in Additional files 3 and 4. Factors included in this analysis were ITN use, anemia, gravidity, transmission season, women's age, the number of IPTp-SP doses, gestational age and malaria status at the $3^{\text {rd }}$ ANC. Only relevant associations are shown as figures in this paper.

Supplemental Tables 3 and 4 present linear regression coefficients of correlation types between ITN use, anemia, gravidity, transmission season, women's age, the number of IPTp-SP doses, gestational age and malaria status at the $3^{\text {rd }} \mathrm{ANC}$ and PM specific antibody types.

ITN users were found to have higher IgG1 levels to DBL5 (Figure 2a; $\mathrm{p}=0.003$ ) and IgG1 levels to ID1-ID2a (Figure 2b; $\mathrm{p}=0.033$ ). The significance of this observation is that higher exposition markers were found in ITN users than non-users.

As expected, multigravida women who probably have experienced malaria during previous pregnancies presented with higher malaria antibody levels than primigravidas. Thus, significantly higher levels DBL5 IgG1 (Figure 3a; $p=0.001$ ), ID1-ID2a total IgG (Figure 3b; $\mathrm{p}=0.021$ ) and ID1-ID2a IgG1 (Figure $3 \mathrm{c} ; \mathrm{p}=0.049$ ) were found.

This study was conducted in an area of intense and seasonal malaria transmission, occurring from July to December, during

Supplemental table 1. Seropositivity to DBL5 and ID1-ID2a at the $3 r d$ ANC visit.

\begin{tabular}{|c|c|c|c|c|c|c|}
\hline \multirow[b]{2}{*}{ Seropositivity } & \multicolumn{2}{|c|}{ IgG to DBL5 } & \multicolumn{2}{|c|}{ IgG1 to DBL5 } & \multicolumn{2}{|c|}{ IgG3 to DBL5 } \\
\hline & Frequency & Percent & Frequency & Percent & Frequency & Percent \\
\hline Negative & 9 & 3.42 & & & 182 & 69.2 \\
\hline \multirow[t]{2}{*}{ Positive } & 254 & 96.58 & 263 & 100 & 81 & 30.8 \\
\hline & IgG to ID1-ID2a & & IgG1 to ID1-ID2a & & \multicolumn{2}{|c|}{ IgG3 to ID1-ID2a } \\
\hline Seropositivity & Frequency & Percent & Frequency & Percent & Frequency & Percent \\
\hline Negative & 14 & 5.32 & & & 136 & 51.71 \\
\hline Positive & 249 & 94.68 & 263 & 100 & 127 & 48.29 \\
\hline
\end{tabular}

Supplemental table 2. Correlation between antibody types. 2a) Spearman's rho (correlation coefficient) 2b) Number of subjects; c: p-value.

\begin{tabular}{|c|c|c|c|c|c|c|}
\hline . & IgG anti-DBL5 & IgG1 anti-DBL5 & IgG3 anti-DBL5 & IgG anti-ID1-ID2a & IgG1 anti-ID1-ID2a & IgG3 anti-ID1-ID2a \\
\hline \multirow[t]{3}{*}{ IgG anti-DBL5 } & 1 & - & - & - & - & - \\
\hline & 263 & & & & & \\
\hline & - & & & & & \\
\hline \multirow[t]{3}{*}{ IgG1 anti-DBL5 } & $0.365^{a}$ & 1 & - & - & - & - \\
\hline & $262^{b}$ & 263 & & & & \\
\hline & $<0.001^{\circ}$ & - & & & & \\
\hline \multirow[t]{3}{*}{ IgG3 anti-DBL5 } & 0.322 & 0.358 & 1 & - & - & - \\
\hline & 263 & 263 & 263 & & & \\
\hline & $<0.001$ & $<0.001$ & - & & & \\
\hline \multirow[t]{3}{*}{ IgG anti-ID1-ID2a } & 0.423 & 0.375 & 0.16 & 1 & - & - \\
\hline & 263 & 263 & 263 & 263 & & \\
\hline & $<0.001$ & $<0.001$ & 0.009 & - & & \\
\hline \multirow[t]{3}{*}{ IgG1 anti-ID1-ID2a } & 0.161 & 0.66 & 0.306 & 0.398 & 1 & - \\
\hline & 263 & 263 & 263 & 263 & 263 & \\
\hline & 0.009 & $<0.001$ & $<0.001$ & $<0.001$ & - & \\
\hline \multirow[t]{3}{*}{ IgG3 anti-ID1-ID2a } & -0.016 & 0.022 & -0.062263 & 0.139 & 0.003 & 1 \\
\hline & 263 & 262 & 0.317 & 263 & 263 & 263 \\
\hline & 0.797 & 0.723 & & 0.024 & 0.957 & - \\
\hline
\end{tabular}


Citation: Traoré O, Benjamin Kaboré WO, Ruizendaal E, Sorgho H, Valéa I, et al. High titres of immunoglobulin g class 1 antibodies to duffy binding-like (dbl) 5 during the third trimester of pregnancy are associated with past placental malaria at delivery in an area of high seasonal transmission in burkina faso. Res Rep Immunol. 2018;2(1):11-2
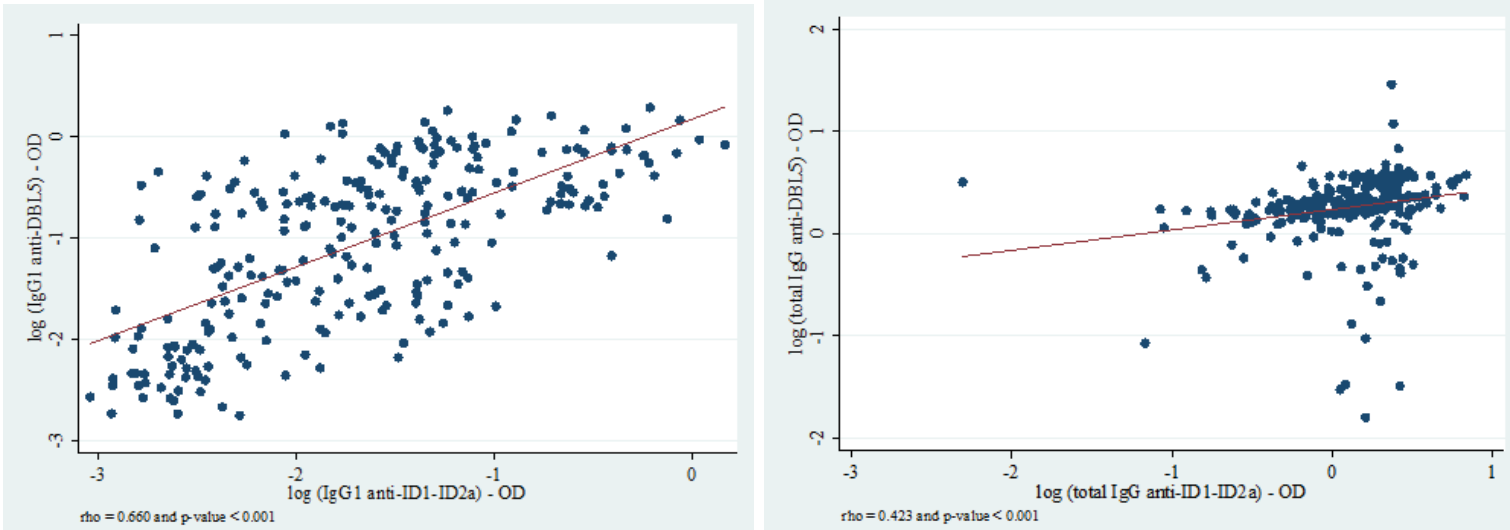

Figure 1. The relationship between titers of antibody types. 1a) Scatter plots showing the moderate correlations between IgG1 anti-DBL5 and IgG1 anti-ID1-ID2a; $1 b) \operatorname{Ig} G$ anti-DBL5 and IgG anti-ID1-ID2a.

Supplemental table 3. Correlation types between general characteristics at the 3rd ANC and antibody levels to DBL5. *1: Multigravidity; *2: trimester; Coef. (95\% CI): Linear regression Coefficient (95\% Confidence Intervals).

\begin{tabular}{|c|c|c|c|c|c|c|c|c|c|c|c|c|}
\hline & \multicolumn{4}{|l|}{$\lg G$} & \multicolumn{4}{|l|}{ IgG1 } & \multicolumn{4}{|l|}{ IgG3 } \\
\hline & Bivariate & & Multivariate & & Bivariate & & Multivariate & & Bivariate & & Multivariate & \\
\hline $\begin{array}{l}\text { General } \\
\text { characteristics }\end{array}$ & Coef. $(95 \% \mathrm{Cl})$ & p & Coef. $(95 \% \mathrm{Cl})$ & p & Coef. $(95 \% \mathrm{Cl})$ & p & Coef. $(95 \% \mathrm{Cl})$ & p & Coef. $(95 \% \mathrm{Cl})$ & p & Coef. $(95 \% \mathrm{Cl})$ & p \\
\hline $\begin{array}{l}\text { Use of ITN } \\
\text { Yes }\end{array}$ & $\begin{array}{c}-0.02 \\
(-0.18-0.14)\end{array}$ & 0.769 & $\begin{array}{c}1 \\
(0.85-1.17)\end{array}$ & 0.987 & $\begin{array}{c}0.19 \\
(0.05-0.33)\end{array}$ & 0.008 & $\begin{array}{c}1.16 \\
(1.01-1.33)\end{array}$ & 0.034 & $\begin{array}{c}0.14 \\
(-0.04-0.32)\end{array}$ & 0.134 & $\begin{array}{c}1.14 \\
(0.95-1.37)\end{array}$ & 0.16 \\
\hline $\begin{array}{l}\text { Anaemia } \\
\text { Anemic }\end{array}$ & $\begin{array}{c}-0.01 \\
(-0.13-0.12) \\
(\end{array}$ & 0.898 & $\begin{array}{c}0.94 \\
(0.84-1.06)\end{array}$ & 0.311 & $\begin{array}{c}0 \\
(-0.10-0.10)\end{array}$ & 0.938 & $\begin{array}{c}1.01 \\
(0.92-1.12)\end{array}$ & 0.78 & $\begin{array}{c}-0.03 \\
(-0.16-0.10)\end{array}$ & 0.649 & $\begin{array}{c}0.96 \\
(0.84-1.10) \\
\end{array}$ & 0.54 \\
\hline $\begin{array}{l}\text { Gravidity } \\
\text { Multi¹. }\end{array}$ & $\begin{array}{c}0.13 \\
(0.00-0.25)\end{array}$ & 0.049 & $\begin{array}{c}1.14 \\
(1.00-1.31)\end{array}$ & 0.053 & $\begin{array}{c}0.17 \\
(0.08-0.27)\end{array}$ & 0.001 & $\begin{array}{c}1.24 \\
(1.10-1.39)\end{array}$ & $<0.001$ & $\begin{array}{c}0.06 \\
(-0.07-0.18)\end{array}$ & 0.391 & $\begin{array}{c}1.01 \\
(0.86-1.17)\end{array}$ & 0.92 \\
\hline $\begin{array}{l}\text { Season of delivery } \\
\text { High }\end{array}$ & $\begin{array}{c}0.09 \\
(-0.01-0.18)\end{array}$ & 0.07 & $\begin{array}{c}1.09 \\
(0.99-1.20)\end{array}$ & 0.087 & $\begin{array}{c}0.04 \\
(-0.03-0.12)\end{array}$ & 0.263 & $\begin{array}{c}1 \\
(0.92-1.08)\end{array}$ & 0.925 & $\begin{array}{c}0.06 \\
(-0.03-0.16)\end{array}$ & 0.192 & $\begin{array}{c}1.04 \\
(0.93-1.17)\end{array}$ & 0.4 \\
\hline $\begin{array}{l}\text { Age } \\
\geq \text { mean }\end{array}$ & $\begin{array}{c}-0.03 \\
(-0.12-0.07) \\
\end{array}$ & 0.564 & $\begin{array}{c}0.95 \\
(0.85-1.05)\end{array}$ & 0.286 & $\begin{array}{c}0.03 \\
(-0.05-0.10)\end{array}$ & 0.47 & $\begin{array}{c}0.94 \\
(0.86-1.03) \\
\end{array}$ & 0.168 & $\begin{array}{c}0.07 \\
(-0.03-0.16)\end{array}$ & 0.167 & $\begin{array}{c}1.07 \\
(0.95-1.20)\end{array}$ & 0.2 \\
\hline $\begin{array}{l}\text { IPTp-SP } \\
>2 \text { doses }\end{array}$ & $\begin{array}{c}0.02 \\
(-0.09-0.12) \\
\end{array}$ & 0.749 & $\begin{array}{c}1.02 \\
(0.91-1.14)\end{array}$ & 0.722 & $\begin{array}{c}-0.16 \\
(-0.25 \text { to }-0.08)\end{array}$ & $<0.001$ & $\begin{array}{c}0.87 \\
(0.79-0.96)\end{array}$ & 0.005 & $\begin{array}{c}-0.1 \\
(-0.21-0.00)\end{array}$ & 0.053 & $\begin{array}{c}0.94 \\
(0.83-1.07)\end{array}$ & 0.37 \\
\hline $\begin{array}{l}\text { Gestational age } \\
\geq 3 \text { rd trim }{ }^{\star} 2 \text {. }\end{array}$ & $\begin{array}{c}0.18 \\
(0.07-0.29)\end{array}$ & 0.001 & $\begin{array}{c}1.17 \\
(1.05-1.30)\end{array}$ & 0.005 & $\begin{array}{c}0.12 \\
(0.03-0.21)\end{array}$ & 0.009 & $\begin{array}{c}1.07 \\
(0.98-1.18)\end{array}$ & 0.133 & $\begin{array}{c}0.09 \\
(-0.02-0.20)\end{array}$ & 0.12 & $\begin{array}{c}1.09 \\
(0.96-1.23)\end{array}$ & 0.17 \\
\hline $\begin{array}{l}\text { Malaria Status at } \\
\text { ANC3 } \\
\text { Infected }\end{array}$ & $\begin{array}{c}0.03 \\
(-0.11-0.17)\end{array}$ & 0.661 & $\begin{array}{c}0.88 \\
(0.76-1.03)\end{array}$ & 0.103 & $\begin{array}{c}0.1 \\
(-0.01-0.21)\end{array}$ & 0.066 & $\begin{array}{c}1.04 \\
(0.92-1.18)\end{array}$ & 0.528 & $\begin{array}{c}0.09 \\
(-0.05-0.23)\end{array}$ & 0.2 & $\begin{array}{c}1.03 \\
(0.87-1.22)\end{array}$ & 0.72 \\
\hline
\end{tabular}

Supplemental table 4. Correlation types between general characteristics at the 3rd ANC and antibody levels to IDI-ID2a.

\begin{tabular}{|c|c|c|c|c|c|c|c|c|c|c|c|c|}
\hline & & $\lg G$ & & & & & lgG1 & & & & G3 & \\
\hline & Bivariate & & Multivariate & & Bivariate & & Multivariate & & Bivariate & & Multivariate & \\
\hline $\begin{array}{l}\text { General } \\
\text { characteristics }\end{array}$ & Coef. $(95 \% \mathrm{Cl})$ & p & $\begin{array}{l}\text { Coef. (95\% } \\
\text { CI) }\end{array}$ & p & $\begin{array}{l}\text { Coef. }(95 \% \\
\text { Cl) }\end{array}$ & $\mathbf{p}$ & Coef. $(95 \% \mathrm{Cl})$ & $\mathbf{p}$ & $\begin{array}{l}\text { Coef. (95\% } \\
\text { CI) }\end{array}$ & $\mathbf{p}$ & $\begin{array}{l}\text { Coef. (95\% } \\
\text { Cl) }\end{array}$ & $\mathbf{p}$ \\
\hline \begin{tabular}{|l|}
$\begin{array}{l}\text { Use of ITN } \\
\text { Yes }\end{array}$ \\
\end{tabular} & $\begin{array}{c}0.13 \\
(-0.04-0.29) \\
\end{array}$ & 0.139 & $\begin{array}{c}1.14 \\
(0.97-1.34) \\
\end{array}$ & 0.105 & $\begin{array}{c}0.1 \\
(0.01-0.20) \\
\end{array}$ & 0.03 & $\begin{array}{c}1.08 \\
(0.98-1.18) \\
\end{array}$ & 0.118 & $\begin{array}{c}-0.04 \\
(-0.21-0.12) \\
\end{array}$ & 0.609 & $\begin{array}{c}0.96 \\
(0.81-1.14) \\
\end{array}$ & 0.635 \\
\hline $\begin{array}{l}\text { Anaemia } \\
\text { Anemic }\end{array}$ & $\begin{array}{c}-0.08 \\
(-0.20-0.04)\end{array}$ & 0.195 & $\begin{array}{c}0.95 \\
(0.84-1.07)\end{array}$ & 0.377 & $\begin{array}{c}0.01 \\
(-0.06-0.07) \\
\end{array}$ & 0.85 & $\begin{array}{c}1.02 \\
(0.95-1.10) \\
\end{array}$ & 0.523 & $\begin{array}{c}0.01 \\
(-0.11-0.13) \\
\end{array}$ & 0.864 & $\begin{array}{c}1.03 \\
(0.91-1.17) \\
\end{array}$ & 0.653 \\
\hline $\begin{array}{l}\text { Gravidity } \\
\text { Multi }{ }^{2} \text {. }\end{array}$ & $\begin{array}{c}0.16 \\
(0.03-0.28) \\
\end{array}$ & 0.014 & $\begin{array}{c}1.11 \\
(0.97-1.26) \\
\end{array}$ & 0.146 & $\begin{array}{c}0.07 \\
(0.00-0.14) \\
\end{array}$ & 0.04 & $\begin{array}{c}1.07 \\
(0.99-1.16) \\
\end{array}$ & 0.086 & $\begin{array}{c}0.08 \\
(-0.04-0.20) \\
\end{array}$ & 0.171 & $\begin{array}{c}1.1 \\
(0.95-1.27) \\
\end{array}$ & 0.202 \\
\hline $\begin{array}{l}\text { Season of } \\
\text { delivery } \\
\text { High }\end{array}$ & $\begin{array}{c}0.23 \\
(0.14-0.32)\end{array}$ & $<0.001$ & $\begin{array}{c}1.25 \\
(1.13-1.38)\end{array}$ & $<0.001$ & $\begin{array}{c}-0.04 \\
(-0.09-0.01)\end{array}$ & 0.15 & $\begin{array}{c}0.95 \\
(0.90-1.01)\end{array}$ & 0.075 & $\begin{array}{c}0.02 \\
(-0.07-0.10)\end{array}$ & 0.718 & $\begin{array}{c}1.02 \\
(0.91-1.13)\end{array}$ & 0.754 \\
\hline \begin{tabular}{|l|} 
Age \\
$\geq$ mean \\
\end{tabular} & $\begin{array}{c}0.08 \\
(-0.01-0.17) \\
\end{array}$ & 0.095 & $\begin{array}{c}1.02 \\
(0.93-1.14)\end{array}$ & 0.636 & $\begin{array}{c}0.03 \\
(-0.02-0.07) \\
\end{array}$ & 0.32 & $\begin{array}{c}0.99 \\
(0.94-1.05) \\
\end{array}$ & 0.84 & $\begin{array}{c}0.03 \\
(-0.06-0.12) \\
\end{array}$ & 0.532 & $\begin{array}{c}1 \\
(0.90-1.12) \\
\end{array}$ & 0.999 \\
\hline $\begin{array}{l}\text { IPTp-SP } \\
>2 \text { doses }\end{array}$ & $\begin{array}{c}-0.12 \\
(-0.23 \text { to }-0.02)\end{array}$ & 0.019 & $\begin{array}{c}1 \\
(0.89-1.12)\end{array}$ & 0.97 & $\begin{array}{l}-0.06 \\
(-0.11 \text { to } \\
-0.00)\end{array}$ & 0.04 & $\begin{array}{c}0.95 \\
(0.88-1.01)\end{array}$ & 0.094 & $\begin{array}{c}0.01 \\
(-0.08-0.11)\end{array}$ & 0.791 & $\begin{array}{c}1.04 \\
(0.92-1.18)\end{array}$ & 0.486 \\
\hline $\begin{array}{l}\text { Gestational } \\
\text { age } \geq 3 \text { rd } \\
\text { trim } * 2 \text {. }\end{array}$ & $\begin{array}{c}0.08 \\
(-0.03-0.19)\end{array}$ & 0.177 & $\begin{array}{c}1.03 \\
(0.92-1.15)\end{array}$ & 0.587 & $\begin{array}{c}0.03 \\
(-0.03-0.09)\end{array}$ & 0.37 & $\begin{array}{c}1.01 \\
(0.95-1.08)\end{array}$ & 0.702 & $\begin{array}{c}0.01 \\
(-0.10-0.11)\end{array}$ & 0.898 & $\begin{array}{c}1.02 \\
(0.90-1.14)\end{array}$ & 0.781 \\
\hline $\begin{array}{l}\text { Malaria Status } \\
\text { at ANC3 } \\
\text { Infected }\end{array}$ & $\begin{array}{c}0.16 \\
(0.03-0.30)\end{array}$ & 0.016 & $\begin{array}{c}1.12 \\
(0.96-1.29)\end{array}$ & 0.146 & $\begin{array}{c}0.06 \\
(-0.01-0.13)\end{array}$ & 0.07 & $\begin{array}{c}1.08 \\
(0.99-1.17)\end{array}$ & 0.094 & $\begin{array}{c}0.01 \\
(-0.12-0.13)\end{array}$ & 0.905 & $\begin{array}{c}1.02 \\
(0.87-1.20)\end{array}$ & 0.762 \\
\hline
\end{tabular}


Citation: Traoré O, Benjamin Kaboré WO, Ruizendaal E, Sorgho H, Valéa I, et al. High titres of immunoglobulin g class 1 antibodies to duffy binding-like (dbl) 5 during the third trimester of pregnancy are associated with past placental malaria at delivery in an area of high seasonal transmission in burkina faso. Res Rep Immunol. 2018;2(1):11-2
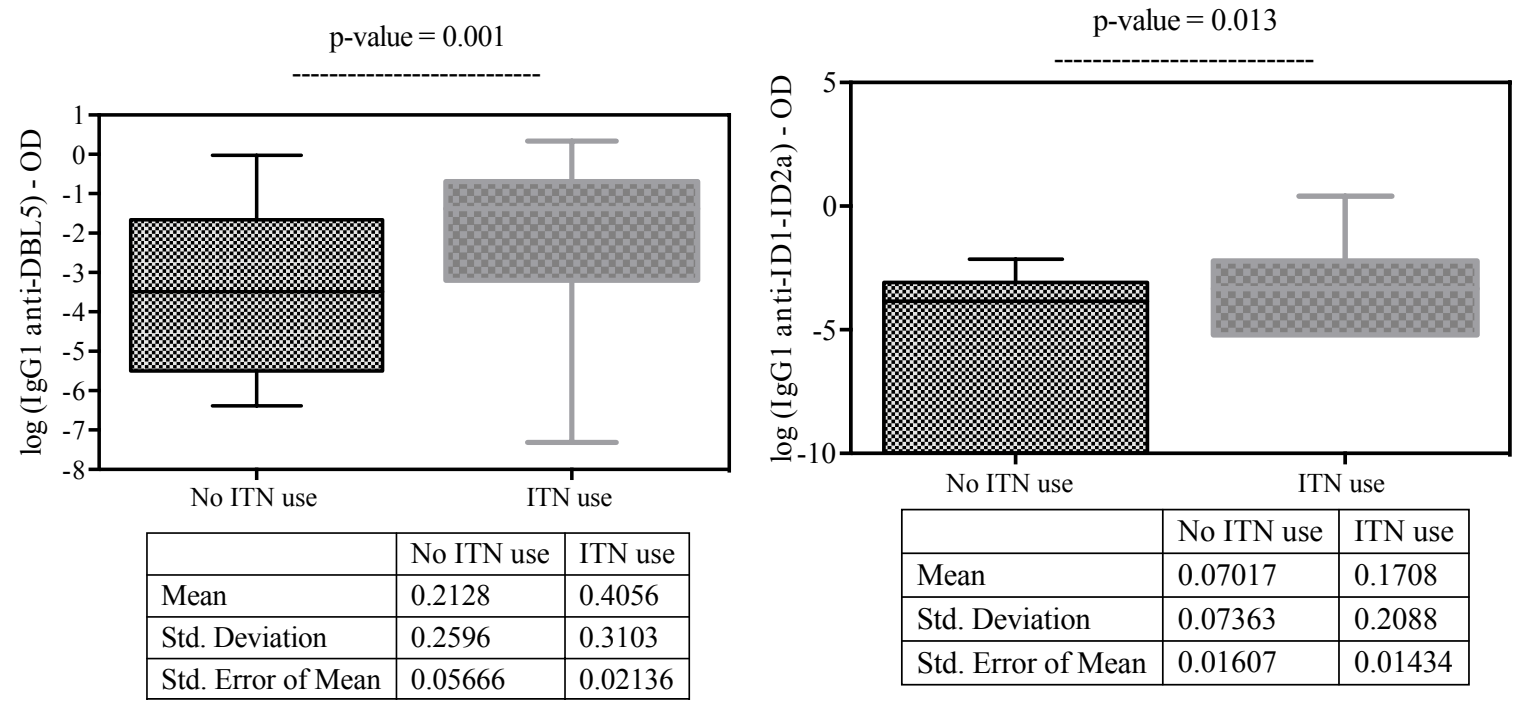

Figure 2. Assessment of the relationship between antibody titers at the $3^{\text {rd }}$ and ITN use. Assessment of the effect of ITN uses on levels of specific antibody to VAR2CSA recombinants fragments. No ITN use $(n=21)$ and ITN use (n=212) 2a) IgG1 levels to DBL5 2; b) IgG1 levels to ID1-ID2a. $P$-value was determined by the Mann-Whitney test.

which, people living in the study area are more exposed to mosquito bites. Pregnant women who performed their ANC 3 visit during this period presented with higher levels of exposition markers. Thus, levels of DBL5 total IgG (Figure 4a; $\mathrm{p}=0.013$ ), ID1-ID2a total IgG (Figure $4 \mathrm{~b} ; \mathrm{p}<0.001$ ) were high in women having per-formed ANC 3 during the high transmission season than those in the low transmission season.

The stratification of antibody levels by the number of IPTpSP doses showed that the uptake of more than two doses was negatively associated with DBL5 IgG1 levels (Figure 5a; $\mathrm{p}=0.032$ ) and IgG3 (Figure. 5b; $\mathrm{p}=0.015$ ). The same effect was also observed with total IgG against ID1-ID2a (Figure 5c; $\mathrm{p}=0.039$ ) when women received more than two doses of IPTpSP. This finding indicates that IPTp given as a full therapeutic course of sulfadoxine-pyrimethamine (SP) and supposed to prevent malaria infection by inducing drug pressure to parasites, has actually induced lower levels of specific antibodies to VAR2CSA antigen (Figure 5d).

When stratifying antibody levels by the age of the pregnancy, it appears that women who performed their ANC 3 visit with higher gestational age had also significantly higher levels of DBL5 total IgG (Figure 6a; $\mathrm{p}<0.001$ ), DBL5 IgG1 (Figure 6b; $\mathrm{p}<0.016)$.

The assessment of the relationship between antibody responses and peripheral malaria infection showed that only ID1-ID2a specific antibody levels were found to be higher in sera samples obtained from women who were seen at the third ANC with peripheral malaria infection [total IgG (Figure $7 \mathrm{a} ; \mathrm{p}=0.014$ ) and IgG1 (Figure 7b; $\mathrm{p}=0.023$ )].

\section{Analysis of the association between factors that influence antibody levels, antibody titers and placental malaria at delivery}

The aim of this study was to analyze the profile of antibody to DBL5 and ID1-ID2a VAR2CSA subdomains during the third trimester of pregnancy and to assess whether these antibodies could predict PM at delivery. The associations between factors that influence antibody levels, antibody titers and PM at delivery are presented in Table 2.

To find the best model, the individual relationships between the characteristic variables, antibody types and PM as outcome, were firstly determined in a bivariate analysis. Our findings showed that titres of DBL5 IgG1 were positively associated with higher risk of $\mathrm{PM}$ at delivery $[\mathrm{OR}=9.545$; $95 \% \mathrm{CI}: 3.45$ 26.40; $<<0.001]$, while the uptake of more than two doses of IPTp-SP [OR $=0.51 ; 95 \%$ CI: $0.27-0.95 ; \mathrm{p}=0.035]$ and the older age $[\mathrm{OR}=0.57 ; 95 \% \mathrm{CI}: 0.33-0.99 ; \mathrm{p}=0.045]$ were associated with reduced risk of PM. Furthermore, ITN use was also associated with higher risk of $\mathrm{PM}[\mathrm{OR}=7.00 ; 95 \% \mathrm{CI}: 1.95$ 25.10; $\mathrm{p}=0.003$.

Secondly, significant associations were included in the multivariate analysis. The multiple logistic regression was used to look for the model that can best explain PM in the study population. In this model, titres of DBL5 $\operatorname{IgG} 1[\mathrm{OR}=11.96$; 95\% CI: 3.66-38.77; $p<0.001]$ and ITN use [OR $=5.17 ; 95 \% \mathrm{CI}$ : 1.36-19.61; $\mathrm{p}=0.016]$ remained positively associated with $\mathrm{PM}$ at delivery, while older women were still at lower risk of PM at delivery [OR=0.45; 95\% CI: 0.23-0.88; $\mathrm{p}=0.021]$.

To further check the type of placental infection that is highly associated with high DBL5 IgG1, antibody levels were stratified by the placenta infection types (Figure 8). Accordingly, high antibody levels were associated with past infections, and the difference between multigravidae and primigravidae with past infection was significant $(\mathrm{p}=0.009)$. Also, in both primigravidae and multigravidae subgroups, women with past PM had higher levels of DBL5 IgG1 compared to those non-infected.

\section{Discussions}

In this study, higher titers of DBL5 IgG1 in the third trimester were significantly associated with placental malaria (PM) at delivery. 
Citation: Traoré O, Benjamin Kaboré WO, Ruizendaal E, Sorgho H, Valéa I, et al. High titres of immunoglobulin g class 1 antibodies to duffy binding-like (dbl) 5 during the third trimester of pregnancy are associated with past placental malaria at delivery in an area of high seasonal transmission in burkina faso. Res Rep Immunol. 2018;2(1):11-2
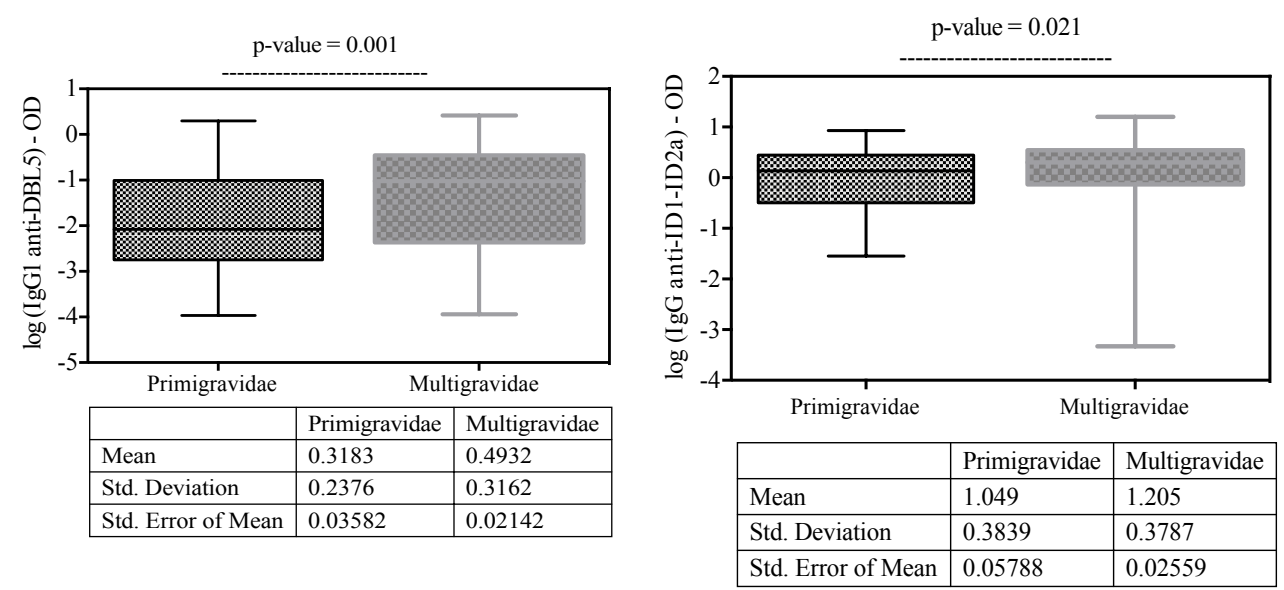

Figure 3. Assessment of the relationship between antibody titers at the $3^{\text {rd }}$ and gravidity. Assessment of the effect of gravidity on levels of specific antibody to VAR2CSA recombinants fragments. Primigravidae $(n=44)$ and Multigravidae $(n=219) 3 a)$ IgG1 levels to DBL5; $3 b)$ total IgG levels to ID1-ID2a; 3c) IgG1 total IgG levels to ID1-ID2a. P-value was determined by the Mann-Whitney test.
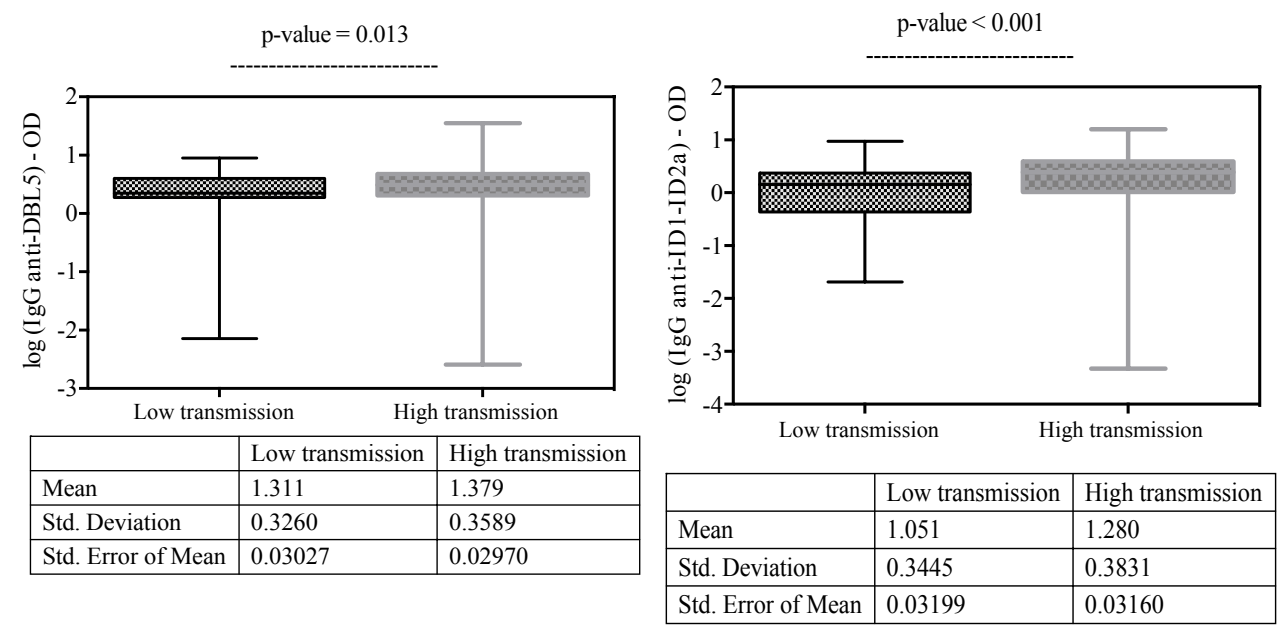

Figure 4: Assessment of the relationship between antibody titers at the $3^{\text {rd }}$ and seasonality. Assessment of the effect of seasonality on levels of specific antibody to VAR2CSA ( $n=116$ for Low transmission season and $n=147$ for High transmission season) $4 a$ ) total IgG levels to DBL5; $4 b)$ total IgG levels to ID1-ID2a. P-value was determined by the Mann-Whitney test.
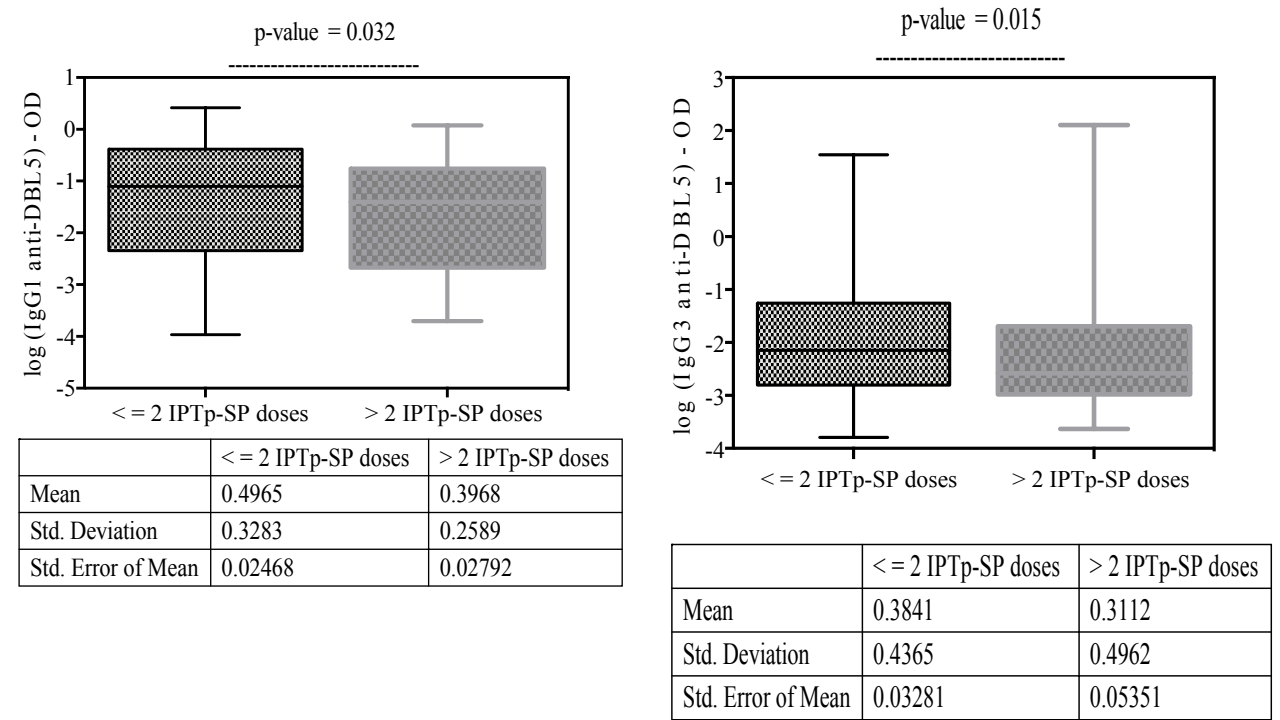

Figure 5. Assessment of the relationship between antibody titers at the $3^{\text {rd }}$ and intermittent preventive treatment. Assessment of the effect of IPTp$S P$ on levels of specific antibody to VAR2CSA recombinants fragments ( $n=177$ for less than 2 doses of IPTp-SP and $n=86$ for more than 2 doses of IPTp-SP) 5 a) IgG1 levels to DBL5; 5b) IgG3 levels to DBL5; 5c) total IgG levels to ID1-ID2a; 5 d) and IgG1 levels to ID1-ID2a. P-value was determined by the Mann-Whitney test. 
Citation: Traoré O, Benjamin Kaboré WO, Ruizendaal E, Sorgho H, Valéa I, et al. High titres of immunoglobulin g class 1 antibodies to duffy binding-like (dbl) 5 during the third trimester of pregnancy are associated with past placental malaria at delivery in an area of high seasonal transmission in burkina faso. Res Rep Immunol. 2018;2(1):11-2
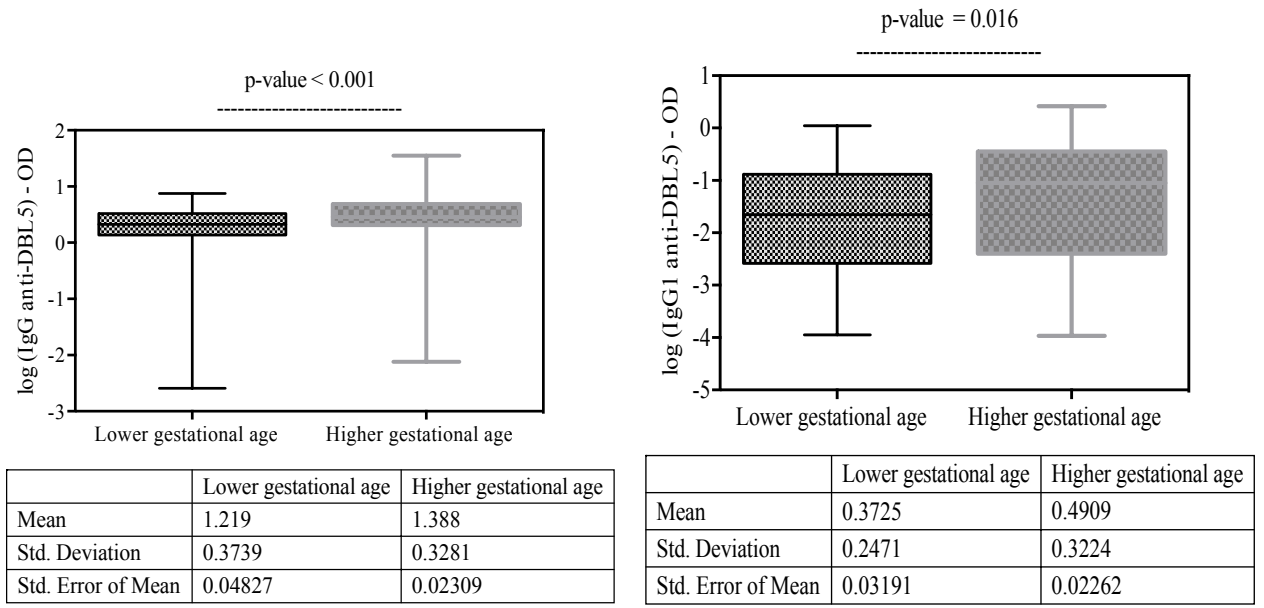

Figure 6. Assessment of the relationship between antibody titers at the 3rd and gestational age. Assessment of the effect of gestational age on levels of specific antibody to VAR2CSA recombinants fragments ( $n=60$ for "Lower gestational age" and $n=203$ for "Higher gestational age") 6 a) total IgG levels to DBL5; 6b) IgG1 levels to DBL5. P-value was determined by the Mann-Whitney test.
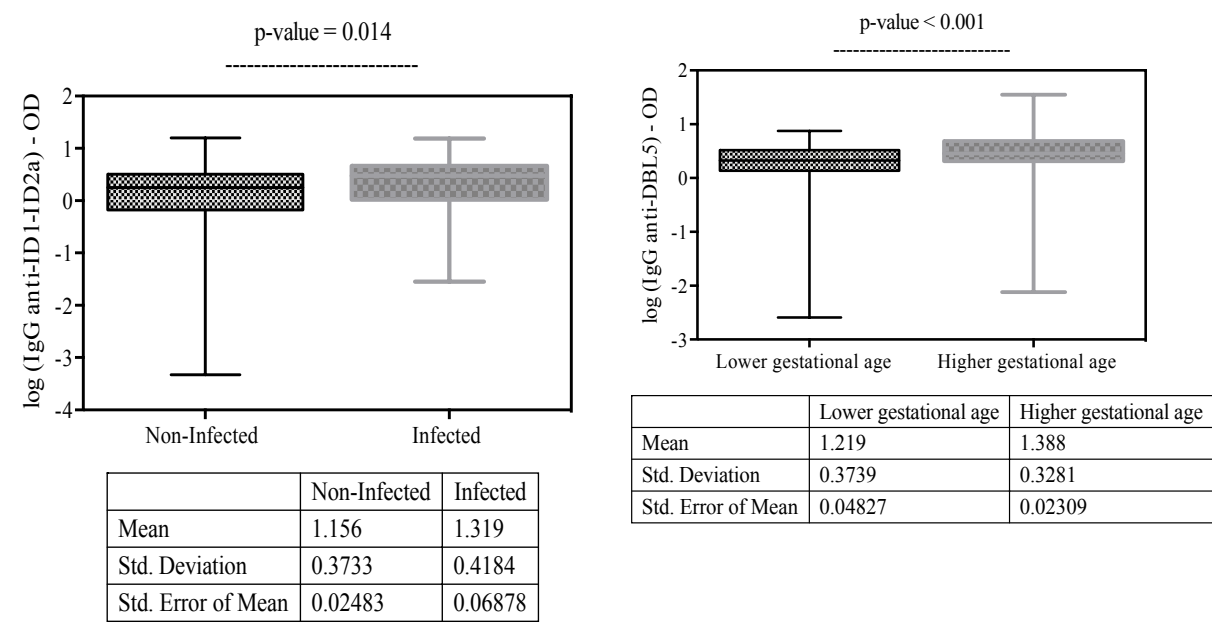

Figure 7. Assessment of the relationship between antibody titers at the $3^{\text {rd }}$ and peripheral malaria infection. Assessment of the effect of peripheral malaria infection on levels of specific antibody to VAR2CSA recombinants fragments. Non-infected ( $n=226)$ and Infected ( $n=37) 7 a)$ total IgG levels to ID1-ID2a; 7b) IgG1 levels to ID1-ID2a. P-value was determined by the Mann-Whitney test.

Table 2. Bivariate and Multivariate analysis assessing factors associated with placental malaria.

\begin{tabular}{|l|c|l|c|l|}
\hline & \multicolumn{2}{|c|}{ Bivariate analysis } & \multicolumn{2}{c|}{ Multivariate analysis } \\
\hline & $\begin{array}{l}\text { Odds ratio (95\% } \\
\text { CI) }\end{array}$ & $\mathbf{p}$ & $\begin{array}{l}\text { Odds ratio (95\% } \\
\text { CI) }\end{array}$ & $p$ \\
\hline $\begin{array}{l}\text { Use of ITN } \\
\text { Yes }\end{array}$ & $\begin{array}{l}7 \\
(1.95-25.10)\end{array}$ & 0.003 & $\begin{array}{c}5.17 \\
(1.36-19.61)\end{array}$ & 0.016 \\
\hline $\begin{array}{l}\text { Anaemia } \\
\text { Anemic }\end{array}$ & $\begin{array}{c}0.85 \\
(0.40-1.79)\end{array}$ & 0.666 & - & - \\
\hline Gravidity & $\begin{array}{l}0.57 \\
(0.26-1.25)\end{array}$ & 0.162 & - & - \\
Multigravidae & $\begin{array}{c}1.03 \\
(0.59-1.78)\end{array}$ & 0.922 & - & - \\
\hline Season of delivery \\
High
\end{tabular}

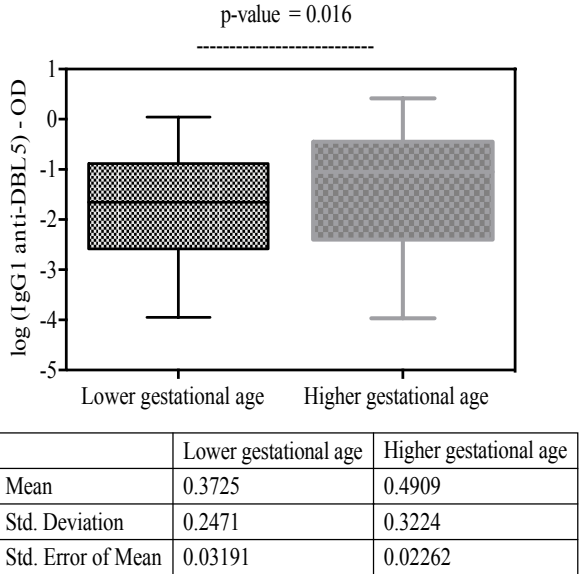


Citation: Traoré O, Benjamin Kaboré WO, Ruizendaal E, Sorgho H, Valéa I, et al. High titres of immunoglobulin g class 1 antibodies to duffy binding-like (dbl) 5 during the third trimester of pregnancy are associated with past placental malaria at delivery in an area of high seasonal transmission in burkina faso. Res Rep Immunol. 2018;2(1):11-2

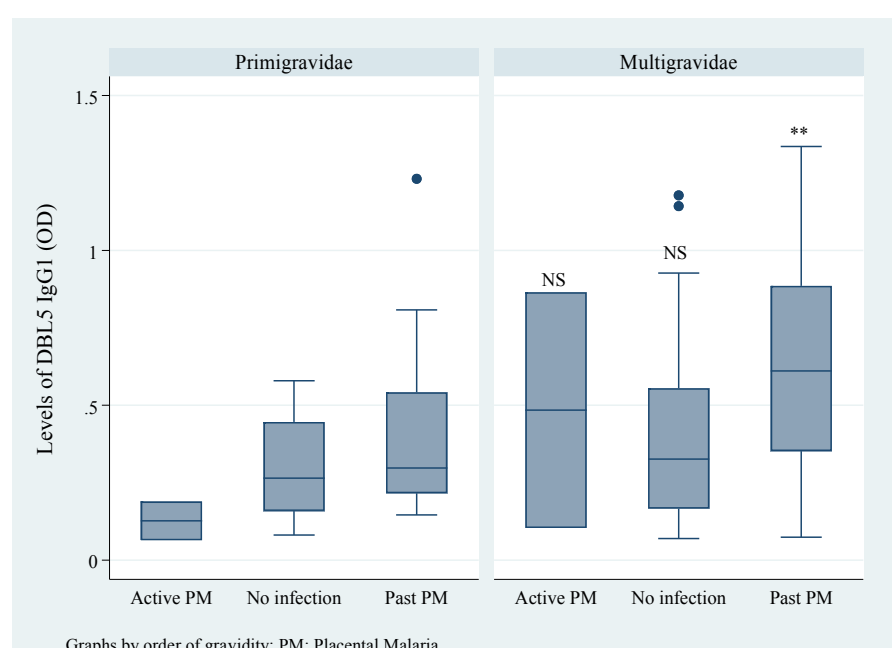

Figure 8. Boxplots illustrating the relationship between antibody titers at the $3^{\text {rd }}$ and placental malaria infection stratified by gravidity. P-values were determined by the Mann-Whitney test; NS: Nonsignificant $p=0.009$.

This association confirms the assumption that DBL5 IgG1 could predict PM at delivery. However, $96.6 \%$ of placental infections detected at delivery were past PM infections i.e. characterized by only the presence of hemozoin in the placenta which indicates an infection occurred earlier during pregnancy [21]. Furthermore, these results could be translated as high levels of DBL5 IgG1 protect [22] against active placental infections, because high levels of this antibody measured at the $3^{\text {rd }}$ trimester, were significantly associated with past placental malaria at delivery. The mechanism of the protection against placental malaria is the capability of specific antibodies to bind to the surfaces of trophozoite-stage infected erythrocytes [23]. Indeed, among antibodies qualified to mediate opsonization of trophozoites in vitro, IgG1 subtype plays a key role [24,25]. Although, neutralizing tests were not performed in the present study, specific naturally acquired antibodies against VAR2CSA recombinant fragments were found in sera samples tested by ELISA. This finding constitutes not only a signal on the fact that PM remains a public health problem [17] in the study area but also an alert on the effectiveness of the application of the national guidelines related to the management of malaria in pregnancy. In the present study, the finding related to the association between ITNs use and high risk of placental malaria infection could have several implications. Firstly, on the immunological basis, the scenario found in this study indicates the inefficiency of ITN use in terms of protection since higher levels of exposition markers (antibodies) have been detected in women using an ITN. This is an indication that pregnant women experienced malaria infection despite the use of ITN. A previous study has actually reported the importance of $\mathrm{P}$. falciparum intensity transmission that significantly influences the acquisition of VSAPAM-specific immunity to pregnancy-associated malaria [16]. Thus, the adequate use of ITN as recommended by the WHO guidelines for preventing malaria during pregnancy in areas with moderate to high transmission [26] should normally reduce the contact human-vector and therefore contributes to downgrade the disease, and therefore induces lower antibody titers. In the present study, data related to the ITN ownership
(93.2\%) and usage (91\%) somewhat collected at the recruitment when gestational age was estimated at least 4 months, constitute rather a proxy for local malaria epidemiology. Secondly, despite the use of ITN reported by women, high antibody levels were found in those having performed ANC 3 visit during the high transmission season, probably they were recruited with preexisting immunity [27] or women may have experienced malaria infection before conception [28] and eventually, residual parasites could have induced later PM resulting in VAR2CSA specific antibody production. Then, if DBL5 IgG1 is produced gradually and even quickly from early pregnancy to the last trimester [29,30], our findings suggest that PM could as well be diagnosed using sera samples at each segment of pregnancy period. The finding related to the downregulation of antibody levels in women receiving more than two doses of IPTpSP, confirmed results from a lower endemicity setting [31]. Even though there is the difference in endemicity between study areas, certainly, it is likely evident that by reducing the risk of infection during pregnancy by regular and adequate uptake of IPTp-SP, both the risk of PM and the production of anti-VAR2CSA antibodies will be reduced. In agreement with several studies [32,33], the present study showed also that older women were at lower risk of PM compared to younger women. Indeed, the majority $(83.3 \%)$ of women in this study were multigravida and mainly adults. Actually, successive pregnancies are supposed to lead women to develop increasing immunity to VAR2CSA producing parasite and reduce the disease frequency and severity [10]. In addition, previous studies that have checked in pregnant women the risk to be infected by malaria during pregnancy, have found that younger women (particularly those aged less than 20 years) independently of parity, were more susceptible than adults [34-36]. Although, for a biomarker to adequately predict a biological event, several competencies including its sensitivity and specificity are needed, in the current study these characteristics were not directly tested. Nevertheless, the approach used in the current study provides valuable information capable to support future investigations. Consequently, these findings suggest that IgG1 anti-DBL5 could be used as a biomarker during pregnancy for the detection of PM cases. Therefore, a diagnostic tool based on IgG1 anti-DBL5, as an optional biomarker, could then be used to guide the clinical management of pregnant women living in high transmission settings until delivery. The current study did not investigate the profile of non-cytophilic antibodies (IgG2 and IgG4) which could have allowed us to assess whether these subtypes of antibodies could predict PM at delivery, and this could be seen as a limitation of the study. However, a previous study has shown that these IgG subtypes do not make up a major component of the $\mathrm{IgG}$ response to placental VSA [22]. Although only two recombinant VAR2CSA domains were used in our study, a recent study carried out in Mali in which eleven VAR2CSA fragments were used has shown that antibody levels vary with P. falciparum parasitemia, gestational age, and gravidity, but do not predict pregnancy outcomes [13]. Another limitation of this study is the fact that antibody levels were measured only during the third trimester when most malaria infection occurs in early pregnancy [37]. Our study raised an important recall on the need to focus on the 
Citation: Traoré O, Benjamin Kaboré WO, Ruizendaal E, Sorgho H, Valéa I, et al. High titres of immunoglobulin g class 1 antibodies to duffy binding-like (dbl) 5 during the third trimester of pregnancy are associated with past placental malaria at delivery in an area of high seasonal transmission in burkina faso. Res Rep Immunol. 2018;2(1):11-2

accurate use of current malaria control tools, and also on the development of new intervention strategies for the management of malaria in pregnancy.

\section{Conclusion}

Our study found a strong association between IgG1 anti-DBL5 during the third trimester of pregnancy and PM at delivery. Therefore, DBL5 specific IgG1 levels as measured during the third trimester could predict PM, that was characterized mostly as past infections. This finding suggests that IgG1 to VAR2CSA could be alternatively used as an optional biomarker to diagnose PM during pregnancy. Such a biomarker could be useful for malaria management in pregnant women in high transmission settings.

\section{Declarations}

\section{Authors'contributions}

OT, ER, and HT conceived and designed the experiments. OT, HS, IV, MT-C, YT, and GAMB supervised the field work. OT and WOBK performed the experiments. OT analyzed the data. OT wrote the manuscript. SS, PF, ER, HS, and UD revised the manuscript. All authors helped to improve the draft, read and approved the final manuscript.

\section{Acknowledgments}

We are grateful to the COSMIC trial participants and the field staff of the Clinical Research Unit of Nanoro for their contribution to the study completion. We would like to thank women (nulliparous) who were recruited in the HDSS catchment area.

\section{Competing interests}

The authors declare that they have no competing interests.

\section{Availability of data and materials}

All data analyzed during this study are included in this published article.

\section{Consent for publication}

Not applicable

\section{Ethics approval and consent to participate}

Informed consent was obtained for each participating community prior to the start of the trial. The study protocol was reviewed and ethically approved by the Institutional Ethics Committee of Center Muraz in Burkina Faso on 19 September 2013 (ref A202013/CE-CM).

\section{Funding}

The COSMIC study was funded by the European Community's Seventh Framework Programme under grant agreement no. 305662 (Project: Community-based scheduled screening and treatment of malaria in pregnancy for improved maternal and infant health: a cluster-randomized trial "COSMIC").

\section{References}

1. WHO. A strategic framework for malaria prevention and control during pregnancy in the African region. Geneva World Health Organization. 2004;1-39.
2. Newman RD. Does WHO support the idea of malaria elimination. Geneva World Health Organization. 2012;1-4.

3. Walker PGT, Floyd J, Kuileter F, et al. Estimated impact on the birth weight of scaling up intermittent preventive treatment of malaria in pregnancy given sulphadoxine-pyrimethamine resistance in Africa: A mathematical model. PLoS Med. 2017;14:1-19.

4. WHO. World Malaria Report 2016. Geneva World Health Organization. 2017:1-186.

5. Fried M, Duffy PE. Malaria during pregnancy. Cold Spring Harb Perspect Med. 2017;7.

6. Mens PF, Bojtor EC, Schallig HDFH. Molecular interactions in the placenta during malaria infection. Eur J Obstet Gynecol Reprod Biol. 2010;152:126-32.

7. Walter PR, Garin Y, Blot P. Placental pathologic changes in malaria. A histologic and ultrastructural study. Am J Pathol. 1982;109:330-42.

8. Achur RN, Valiyaveettil M, Alkhalil A, et al. Characterization of proteoglycans of human placenta and identification of unique chondroitin sulfate proteoglycans of the intervillous spaces that mediate the adherence of Plasmodium falciparuminfected erythrocytes to the placenta. J Biol Chem. 2000;275:40344-56.

9. Beeson JG, Rogerson SJ, Cooke BM, et al. Adhesion of Plasmodium falciparum-infected erythrocytes to hyaluronic acid in placental malaria. Nat Med. 2000;6:86-90.

10. Fried M, Duffy PE. Adherence of Plasmodium falciparum to chondroitin sulfate $\mathrm{A}$ in the human placenta. Science. 1996;272:1502-4.

11. Salanti A, Dahlbäck M, Turner L, et al. Evidence for the involvement of VAR2CSA in pregnancy-associated malaria. J Exp Med. 2004;200:1197-203.

12. Dechavanne S, Srivastava A, Gangnard S, et al. Paritydependent recognition of DBL1X-3X suggests an important role of the VAR2CSA high-affinity CSAbinding region in the development of the humoral response against placental malaria. Infect Immun. 2015;83:2466-74.

13. Fried M, Kurtis JD, Swihart B, et al. Antibody levels to recombinant VAR2CSA domains vary with Plasmodium falciparum parasitemia, gestational age, and gravidity, but do not predict pregnancy outcomes. Malar J. 2018;17:106.

14. Fried M, Duffy PE. Designing a VAR2CSA-based vaccine to prevent placental malaria. Vaccine. 2015;33:7483-8

15. Gangnard S, Tuikue Ndam NG, Gnidehou S, et al. Functional and immunological characterization of the var2CSA-DBL5 $\varepsilon$ domain of a placental Plasmodium 
Citation: Traoré O, Benjamin Kaboré WO, Ruizendaal E, Sorgho H, Valéa I, et al. High titres of immunoglobulin g class 1 antibodies to duffy binding-like (dbl) 5 during the third trimester of pregnancy are associated with past placental malaria at delivery in an area of high seasonal transmission in burkina faso. Res Rep Immunol. 2018;2(1):11-2

falciparum isolate. Mol Biochem Parasitol. 2010;173:115-22.

16. Megnekou R, Staalsoe T, Taylor DW, et al. Effects of pregnancy and intensity of Plasmodium falciparum transmission on immunoglobulin $\mathrm{G}$ subclass responses to variant surface antigens. Infect Immun. 2005;73:4112-8.

17. Natama HM, Ouedraogo DF, Sorgho $H$, et al. Diagnosing congenital malaria in a high-transmission setting: clinical relevance and usefulness of $P$. falciparum HRP2-based testing. Sci Rep. 2017;7:1279.

18. Scott S, Mens PF, Tinto H, et al. Community-based scheduled screening and treatment of malaria in pregnancy for improved maternal and infant health in The Gambia, Burkina Faso, and Benin: study protocol for a randomized controlled trial. Trials. 2014;15:2603.

19. Clausen TM, Christoffersen S, Dahlback M, et al. Structural and functional insight into how the Plasmodium falciparum VAR2CSA protein mediates binding to chondroitin sulfate A in placental malaria. $\mathrm{J}$ Biol Chem. 2012;287:23332-45.

20. Guitard J, Cottrell G, Magnouha NM, et al. Differential evolution of anti-VAR2CSA- IgG3 in primigravidae and multigravidae pregnant women infected by Plasmodium falciparum. Malar J. 2008;7:10.

21. Bulmer JN, Rasheed FN, Francis N, et al. Placental malaria I. Pathological classification. Histopathology. 1993;22:211-8.

22. Elliott SR, Brennan AK, Beeson JG, et al. Placental malaria induces variant-specific antibodies of the cytophilic subtypes immunoglobulin G1 (IgG1) and IgG3 that correlate with adhesion inhibitory activity. Infect Immun. 2005;73:5903-7.

23. Piper KP, Roberts DJ, Day KP. Plasmodium falciparum: analysis of the antibody specificity to the surface of the trophozoite-infected erythrocyte. Exp Parasitol. 1999;91:161-9.

24. Groux H, Gysin J. Opsonization as an effector mechanism in human protection against asexual blood stages of Plasmodium falciparum: functional role of IgG subclasses. Res Immunol. 1990;141:529-42.

25. Tebo AE, Kremsner PG, Luty AJF. Fcgamma receptormediated phagocytosis of Plasmodium falciparuminfected erythrocytes in vitro. Clin Exp Immunol. 2002;130:300-6.

26. Tako EA, Zhou A, Lohoue J, et al. Risk factors for placental malaria and its effect on pregnancy outcome in Yaounde, Cameroon. Am J Trop Med Hyg. 2005;72:236-42.

27. Ofori MF, Lamptey H, Dickson EK, et al. Etiology of placental Plasmodium falciparum malaria in African women. J Infect Dis. 2018;32:157-5.
28. Tuikue Ndam N, Tornyigah B, Dossou AY, et al Persistent Plasmodium falciparum infection in women with intent to become pregnant is a risk factor for pregnancy-associated malaria. Clin Infect Dis. 2018.

29. Jefferis R, Kumararatne DS. Selective IgG subclass deficiency: quantification and clinical relevance. Clin Exp Immunol. 1990;81:357-67.

30. Tutterrow YL, Avril M, Singh K, et al. High levels of antibodies to multiple domains and strains of VAR2CSA correlate with the absence of placental malaria in Cameroonian women living in an area of high Plasmodium falciparum transmission. Infect Immun. 2012;80:1479-90.

31. Staalsoe T, Shulman CE, Dorman EK, et al. Intermittent preventive sulfadoxine-pyrimethamine treatment of primigravidae reduces levels of plasma immunoglobulin $\mathrm{G}$, which protects against pregnancyassociated Plasmodium falciparum malaria. Infect Immun. 2004;72:5027-30.

32. Snow RW, Guyatt HL. The epidemiology and burden of Plasmodium falciparum-related anemia among pregnant women in sub-Saharan Africa. Am J Trop Med Hyg. 2001;64:36-44.

33. Shulman CE, Marshall T, Dorman EK, et al. Malaria in pregnancy: adverse effects on hemoglobin levels and birthweight in primigravidae and multigravidae. Trop Med Int Health. 2001;6:770-8.

34. Poespoprodjo JR, Fobia W, Kenangalem E, et al. Adverse pregnancy outcomes in an area where multidrug-resistant Plasmodium vivax and Plasmodium falciparum infections are endemic. Clin Infect Dis. 2008;46:1374-81.

35. Hamer DH, Singh MP, Wylie BJ, et al. Burden of malaria in pregnancy in Jharkhand State, India. Malar J. 2009;8:210.

36. Ayoola OO, Whatmore A, Balogun WO, et al. Maternal malaria status and metabolic profiles in pregnancy and in cord blood: relationships with birth size in Nigerian infants. Malar J. 2012;11:75.

37. Huynh BT, Cottrell G, Cot M, et al. Burden of malaria in early pregnancy: a neglected problem? Clin Infect Dis. 2015;60:598-604.

\author{
*Correspondence to: \\ Ousmane Traoré \\ Clinical Research Unit of Nanoro (IRSS-URCN) \\ Institute of Research in Health Sciences, Nanoro, \\ Burkina Faso \\ E-mail: ousmane_tra@yahoo.fr
}

